\title{
Diagnostic Accuracy of Contrast Transthoracic Echocardiography, Contrast Transcranial Doppler and Transesophageal Echocardiography in Patients Undergoing Patent Foramen Ovale Closure
}

\section{Kaijing Wang}

The First Affiliated Hospital of Wenzhou Medical University

Xianda Ni

The First Affiliated Hospital of Wenzhou Medical University

Yuxin Wu

Wenzhou Medical University

Chen Chen ( $\nabla$ chenchenowen@126.com)

The First Affiliated Hospital of Wenzhou Medical University

\section{Research Article}

Keywords: patent foramen ovale, TEE, CTTE, CTCD, closure

Posted Date: May 19th, 2021

DOl: https://doi.org/10.21203/rs.3.rs-440132/v1

License: (c) (1) This work is licensed under a Creative Commons Attribution 4.0 International License.

Read Full License 


\section{Abstract}

Purpose: To compare diagnostic accuracy of CTTE, CTCD and TEE in patients undergoing patent foramen ovale closure (PFO) so as to provide evidence for clinical decision-making.

Methods: The clinical data of 140 patients with cerebral infarction or migraine who successfully underwent PFO closure or whose right cardiac catheterization results showed no PFO in our hospital were analyzed retrospectively, including clinical baseline, data of CTTE, CTCD and TEE.

Results: Patients who successfully underwent PFO closure or whose right cardiac catheterization results showed no PFO were divided into group A and group B, respectively. The differences of the shunt in cTTE and CTCD, the diameter of PFO in TEE and the appearance of bubbles in CTTE were compared. The results showed that the proportion of medium-large shunt in group A was significantly higher than group $B$ in resting CTCD, post-Valsalva cTCD and total CTCD respectively (67.35\% VS $42.86 \% \bigotimes P=0.004 \unrhd 100 \%$ VS $71.42 \% \otimes \mathrm{P}=0.0003 \otimes 81.63 \% \mathrm{VS} 51.43 \% \otimes \mathrm{P}=0.002$ ). The proportion of medium-large shunt in group $A$ was higher than group B in CTCD (75.90\% VS 19.35\%खP 0.001$)$. The diameters of PFO of group A were larger than group $B$ in TEE $(2.18 \pm 0.78$ VS 1.19 $\pm 0.78 \otimes P \llbracket 0.001)$. Take the successful PFO closure as the state variable. The ROC curves of CTTE, CTCD and TEE were made, and the results showed that CTCD and TEE had better sensitivity and specificity. The proportion of appearance of bubbles within 5 cardiac cycles in CTTE in group A was significantly higher than group $B(73.47 \%$ VS $42.86 \% \otimes P=0.005)$.

Conclusions: For the patients with PFO to be occluded, the success rate of operation could be improved by selecting patients with larger shunt, larger diameter of foramen ovale and shorter interval in the appearance of bubbles. Otherwise, there was perhaps no PFO. CTCD and TEE had better diagnostic value for PFO closure than that of cTTE.

\section{Introduction}

The patent foramen ovale (PFO) is a small opening in the wall between the right and left upper chambers of the atria. Thrombus from the vein can enter the systemic circulation through this opening by right-toleft shunt (RLS) to cause paradoxical embolization. Epidemiological studies indicated that the morbidity was about 9.2\% [1]. In recent years, more and more attention has been paid to PFO. It has been confirmed that PFO is related to paradoxical embolism, including cryptogenic stroke(CS) and transient ischemic attack(TIA) [2, 3]. Migraine was also considered to be closely related to PFO $[4,5]$.

At present, PFO closure was considered to be an effective measure to prevent CS and migraine, and the effect was better than drug [6]. The guidelines of the United States and Europe both recommend PFO closure for indicated patients.

For the patients who were clinically suspected of CS or migraine and considered to be suitable for PFO closure, the guidelines recommended contrast transthoracic echocardiography (cTTE), transesophageal 
echocardiography (TEE) and contrast transcranial Doppler (CTCD) to verify the existence of RLS and serve as a basis for the diagnosis of CS or migraine [7].

Although some patients highly suspected of CS or migraine in clinic and CTTE, TEE, CTCD also indicated RLS, the PFO closure could not be completed in many cases because of the guide wire could not pass through the foramen ovale.

This study will further explore the clinical characteristics of the cases with cerebral infarction or migraine who successfully underwent PFO closure and whose right cardiac catheterization results showed no PFO, so as to help clinicians make better clinical decisions, better screen patients, and better communicate with them.

\section{Materials And Methods}

\section{Study population}

Our study was approved by the ethic committee of the First Affiliated Hospital of Wenzhou Medical University. In this study, we retrospectively analyzed 140 patients who underwent PFO closure or right cardiac catheterization in the Department of Cardiology of The First Affiliated Hospital of Wenzhou Medical University from August, 2018 to December, 2020. 97 cases successfully underwent PFO closure and 43 cases did not have PFO based on the results of the right cardiac catheterization; 37 cases of cerebral infarction and 103 cases of migraine; 84 cases underwent CTTE, 130 cases underwent TEE and 114 cases underwent CTCD. All patients were examined by routine blood tests, Holter, head CT or magnetic resonance, vascular ultrasound, etc. after admission.

The definition and grading of CTTE

The standard apical four-chamber view was taken by GE vividE95 Doppler echocardiography. After intravenous injection of activated saline, the microbubble signal in the left ventricular system was defined as positive within 3-5 cardiac cycles after the right heart system was filled with microbubble signal. Then the activated saline was injected intravenously again after Valsalva maneuver. Grading standard [8]: grade 0 , no microbubble; grade I, mild RLS, 1-10 microbubbles; grade II, moderate RLS, 11-30 microbubbles; grade III, massive RLS, $>30$ microbubbles or the left atrium was almost full of microbubbles (Figure 1).

The definition and grading of CTCD

Elica transcranial Doppler detector with probe frequency of $1.6 \mathrm{MHz}$ was used to monitor the left middle cerebral artery. The number of microbubbles in the middle cerebral artery was observed within 10 seconds after intravenous injection of activated saline. If the microbubble signal was not detected in the resting state, the activated saline was injected intravenously again after Valsalva maneuver. Grading standard [9]: grade 0, no shunt, no microbubble; grade I, mild shunt, 1-10 microbubbles; grade II, moderate shunt, 10-25 microbubbles; grade III, massive shunt, >25 microbubbles. 
The clinician inserted the probe through the esophagus and scanned in the direction of $0^{\circ}-180^{\circ}$ to show the complete atrial septum. In the sections of two atriums and the superior and inferior vena cava of both atriums, we focused on whether there was a gap between the primary secondary septum. Then we measured the internal diameter of the gap and observed whether there was oblique transseptal shunt with Doppler flow echo (Figure 2).

Indications and contraindication of PFO closure[7]

Indications: (1) Stroke of unknown causes or TIA with PFO, had moderate or massive RLS, or relapsed with anticoagulant therapy, or had definite deep venous thrombosis. (2) Intractable or chronic migraine complicated with moderate or massive RLS in PFO. (3) PFO complicated with venous thrombosis or varicose veins / valvular insufficiency of lower extremities, with moderate or massive RLS. (4) High risk PFO, PFO complicated with atrial septal neoplasm, large diameter or resting RLS. (5) The age was from 18 to 60 years old.

Relative indications: (1) PFO with migraine; (2) PFO with high risk factors of venous thrombosis (longterm sitting or lying in bed); (3) PFO with extracranial artery embolism; (4) special occupations with PFO (such as divers, etc.); (5) hypoxia with PFO which was difficult to explain clinically.

Contraindication: (1) cerebral embolism of any cause could be found; (2) could not tolerate antiplatelet or anticoagulant therapy; (3) thrombosis of inferior vena cava or pelvic vein led to complete obstruction, systemic or local infection, septicemia, and intracardiac thrombosis. (4) pregnancy; (5) complicated with pulmonary hypertension or PFO as a special channel; (6) acute stroke in 2 weeks.

The procedure of PFO closure

The PFO occluder made by Shanghai Shape Memory Alloy Co., Ltd. was selected. After femoral vein puncture, we sent the guide wire and $6 \mathrm{~F}$ right heart catheter to the right atrium under $\mathrm{X}$-ray. Then the guide wire and catheter were sent into the left superior pulmonary vein through the foramen ovale. Next, we replaced guide wire with super stiff wire and sent the sheath into the left atrium. At last, we sent into the PFO occluder and released it after the position was confirmed by X-ray and ultrasound. After operation, aspirin was given orally $100 \mathrm{mg}$ once a day for 6 months.

Statistical analysis

SPSS20.0 software was used for statistical analysis. All measurement data were expressed as mean \pm standard deviation, and the differences between groups were analyzed by $T$ test. All counting data were expressed by constituent ratio. The differences between groups were analyzed by Mann-Whitney $U$ test. The sensitivity and specificity of the method were evaluated by ROC curve. $P<0.05$ was considered statistically significant. 


\section{Results}

A total of 140 patients were enrolled, of which 97 cases successfully underwent PFO closure in group A, and 43 cases did not have PFO based on the results of the right cardiac catheterization in group $\mathrm{B}$. The difference of clinical baseline data between the two groups was analyzed (Table 1). The results showed that there was no significant difference in age, gender, hypertension, diabetes, smoking and stoke/ migraine between the two groups.

Table 1 Comparison of clinical baseline data between patients successfully underwent PFO closure or not have PFO based on the results of the right cardiac catheterization

\begin{tabular}{|llll|}
\hline & Group A (N=97) & Group B (N=43) & $P$ \\
\hline Age, years & $46.01 \pm 12.37$ & $43.28 \pm 11.77$ & 0.22 \\
\hline Gender (\%) & & & 0.19 \\
\hline Male & $43(44.33)$ & $14(32.56)$ & \\
\hline Female & $54(55.67)$ & $29(67.44)$ & \\
\hline Hypertension (\%) & & & 0.50 \\
\hline Yes & $23(23.71)$ & $8(18.60)$ & \\
\hline No & $74(76.29)$ & $35(81.40)$ & \\
\hline Diabetes (\%) & & & 0.88 \\
\hline Yes & $4(4.12)$ & $2(4.65)$ & \\
\hline No & $93(95.88)$ & $41(95.35)$ & \\
\hline Smoking (\%) & & & \\
\hline Yes & $22(22.68)$ & $6(13.95)$ & \\
\hline No & $75(77.32)$ & $37(86.05)$ & \\
\hline Stroke/Migraine (\%) & & & \\
\hline Yes & $29(29.90)$ & $8(18.60)$ & \\
\hline No & $68(70.10)$ & $35(81.40)$ & \\
\hline
\end{tabular}

The differences of the shunt in CTTE and CTCD, the diameter of PFO in TEE and the appearance of bubbles in cTTE between group A and group B were compared (Table 2). The cTTE score was divided into 
resting score, post-Valsalva score and total score. The total score was the sum of the first two scores, and the corresponding score was taken according to the shunt grade. The results suggested that the proportion of medium-massive shunt in group A was higher than that in group B in cTTE. In resting state, medium-massive shunt (2-3 points) in group A accounted for $67.35 \%$, while that in group B accounted for $42.86 \%$. The difference was statistically significant $(P=0.004)$. After Valsalva maneuver, medium-large shunt (2-3 points) in group $A$ accounted for $100 \%$, and $71.42 \%$ in group $B$, and the difference was statistically significant $(P=0.0003)$. In total score, medium-massive shunt (4-6 points) in group $A$ accounted for $81.63 \%$, and $51.43 \%$ in group $B$. The difference was also statistically significant $(P=0.002)$. The corresponding score of CTCD was taken according to the shunt grade. In CTCD, the proportion of medium-massive shunt (2-3 points) accounted for $75.90 \%$ and $19.35 \%$ in group $A$ and group $B$, respectively. The difference was statistically significant $(P<0.001)$. In TEE, the PFO diameters in group $A$ $(2.18 \pm 0.78)$ was larger than that in group $B(1.19 \pm 0.78)$. The difference was statistically significant $(P<$ 0.001). In terms of the appearance of bubbles in CTTE, $73.47 \%$ of group A was less than 5 cardiac cycles and the number for group $B$ was $42.86 \%$, showing the statistically significant difference $(P=0.005)$.

\section{Table2 Comparison of cTTE, CTCD, TEE and the appearance of bubbles between patients successfully undrwent PFO closure or not have PFO based on the results of the right cardiac catheterization}

\begin{tabular}{|c|c|c|c|}
\hline & Group A & Group B & $P$ \\
\hline \multicolumn{4}{|l|}{ cTTE } \\
\hline Resting (point, \%) & $2-3 \rrbracket 67.35 \rrbracket$ & $2-3 \varangle 42.86 \rrbracket$ & 0.004 \\
\hline Post-Valsalva (point, \%) & $2-3 \otimes 100.00 \rrbracket$ & $2-3 \otimes 71.43 \rrbracket$ & 0.003 \\
\hline Total (point, \%) & $4-6 \rrbracket 81.63 \rrbracket$ & $4-6 \rrbracket 51.43 \rrbracket$ & 0.002 \\
\hline cTCD (point, \%) & $2-3 \otimes 75.90 \otimes$ & $2-3 \otimes 19.35 \rrbracket$ & $\bowtie 0.001$ \\
\hline TEE (mm) & $2.18 \pm 0.78$ & $1.19 \pm 0.78$ & $\bowtie 0.001$ \\
\hline Appearance of bubbles less than 5 cardiac cycles (case, \%) & $36 \rrbracket 73.47 \% \rrbracket$ & $15 \llbracket 42.86 \% \rrbracket$ & 0.005 \\
\hline
\end{tabular}

Take the successful PFO closure as the state variable. The ROC curves of CTTE, CTCD and TEE were made (Figure 3). The results showed that the areas under the ROC curve (AUC) of CTCD and TEE were larger and had better sensitivity and specificity (Table 3 ). The AUC of cTCD was $0.823(P<0.001)$, the sensitivity was 0.800 and the specificity was 0.769 . The AUC of TEE was $0.785(P<0.001)$, the sensitivity was 0.850 and the specificity was 0.692 . The AUC of resting CTTE was 0.638 , the sensitivity was 0.925 , and the specificity was 0.308 . The AUC of post-Valsalva cTTE was 0.667 , the sensitivity was 1.000 , and the specificity was 0.308 . The AUC of CTTE was 0.665 , the sensitivity was 0.975 , and the specificity was 0.423 . 
Table 3 Comparison of sensitivity and specificity in cTTE, cTCD and TEE

\begin{tabular}{|c|c|c|c|c|c|c|}
\hline & & AUC & $P$ & Sensitivity & Specificity & Jordan index \\
\hline CTCD & & 0.823 & $<0.001$ & 0.800 & 0.769 & 1.5 \\
\hline TEE & & 0.785 & $<0.001$ & 0.850 & 0.692 & 1.7 \\
\hline \multirow[t]{3}{*}{ cTTE } & Resting & 0.638 & 0.058 & 0.925 & 0.308 & 1.5 \\
\hline & Post-Valsalva & 0.667 & 0.022 & 1.000 & 0.308 & 1.5 \\
\hline & Total & 0.665 & 0.024 & 0.975 & 0.423 & 2.5 \\
\hline
\end{tabular}

\section{Discussion}

PFO closure is a rapidly developing interventional technique in recent years. With the further understanding of the causes of CS and migraine, PFO closure has been further developed. At present, it is considered that PFO closure can prevent CS and TIA events, and the effect is better than drug therapy. Although the procedure of PFO closure is similar to that of transcatheter closure of atrial septal defect, it has its own difficulty. It is relatively difficult for the catheter to pass through the foramen ovale. At present, there is little literature concerning the discussion of the clinical difference between patients who successfully underwent PFO closure and whose right cardiac catheterization results showed no PFO. This study bridged the gap by reviewing 140 patients of the two types mentioned above. The results suggest that: (1) the level of shunt in CTTE and CTCD was higher in patients with successful closure, and the diameter of foramen ovale in TEE was larger in patients with successful closure. (2) CTCD and TEE had high sensitivity and specificity in diagnosis of PFO. The sensitivity of CTTE was too high and led to many false positive cases. So, the diagnostic value of CTCD and TEE was better than CTTE. (3) In patients without PFO, the bubbles could appear after 5 cardiac cycles instead of within 5 cardiac cycles in cTTE, suggesting that there might be no PFO in those patients. Other RLS diseases such as pulmonary arteriovenous fistula (PAF) should be considered.

At present, CTTE and CTCD were routine tests for screening PFO. The latest study showed that the sensitivity and specificity of single CTTE or CTCD for the diagnosis of PFO were satisfactory when the cutoff of CTTE and CTCD were taken in massive shunt. But the combination of the two tests could further improve the sensitivity and specificity [10]. Other studies also believed that both CTTE and CTCD could be used for the screening of PFO. The semi-quantitative grading of CTCD and CTTE were related to the diameter of PFO, and the diameter of PFO with higher grade was larger [11]. The conclusion of our study was similar to those results. Shunt grades of successful PFO closure were higher in CTTE and cTCD, and the diameter of foramen ovale in TEE was larger. It was suggested that when screening patients in clinic, the patients who had medium-large shunt in both CTTE and CTCD, and with larger foramen ovale in TEE could have higher success rate of PFO closure. This study set the success of PFO closure, in other words, 
whether the guide wire could pass through the foramen ovale, as the gold standard. However, most previous studies considered TEE as the gold standard to evaluate CTTE and CTCD. So, our study could provide a direct evidence for PFO closure.

This study further compared the specificity and sensitivity of CTTE, TEE and CTCD. Moreover, the success of PFO closure was considered as the gold standard, which was not reported in previous studies. The results suggested that CTCD and TEE have higher diagnostic value in PFO, and was superior to CTTE. One study has shown that after adequate Valsalva manoeuvre, CTTE was very sensitive to PFO and could be used as a routine screening method for PFO. While TEE was less sensitive to PFO because Valsalva manoeuvre was not adequately performed on patients. So it was less sensitive than cTTE in PFO [12]. The latest study also believed that adequate Valsalva manoeuvre could improve the sensitivity of cTTE [13]. The results of our study were similar to the above results. cTTE had a very high sensitivity which was higher than TEE and CTCD. The reason might be that the Valsalva manoeuvre was more adequately performed on patients in CTTE, leading to the increase of the right heart pressure. The foramen ovale could be temporary opening, which led to false positive cases. In our study, it was found that although TEE and CTCD had slightly lower sensitivity than CTTE, the specificity was satisfactory. Hence, the comprehensive diagnostic value of TEE and CTCD was higher than CTTE. TEE and CTCD could better predict the outcome of PFO closure. Therefore, for patients who are going to undergo PFO closure, all the three examinations should be performed to evaluate the success rate of PFO closure.

Pulmonary arteriovenous fistula (PAF) could also lead to paradoxical embolization. Delayed left ventricular enhancement in CTTE was one of the characteristics of PAF and one of the evidences for differentiating the source of shunt. In general, the shunt came from PFO if left ventricle was enhanced within 3 to 5 cardiac cycles. While it was considered that the shunt came from PAF if left ventricle was enhanced in more than 5 cardiac cycles [14]. In our study, the number of patients whose appearance of bubbles within 5 cardiac cycles in cTTE in group A was significantly larger than those in group $B$, and one patient was confirmed with PAF by angiography. Therefore, for the patients whose right cardiac catheterization results show no PFO, we should conduct further examination to screen other diseases that could cause paradoxical embolization, such as PAF.

This study was a single-center, retrospective study. We look forward to the results of multicenter, prospective studies in the future, which could provide further evidence for PFO closure. In addition, the sample size of this study was still limited, and larger sample could be used in future studies.

To sum up, this study found that patients with successful PFO closure had larger shunts in CTTE and CTCD, larger foramen ovale diameter in TEE, and a shorter interval in the appearance of bubbles in cTTE. It was suggested that among the patients undergoing PFO closure, the patients with larger shunt, larger diameter of foramen ovale and shorter interval in the appearance of bubbles could improve the success rate of PFO closure. On the contrary, there was a decrease in the probability of PFO. Moreover, further screening should be performed to explore other causes of CS or migraine. CTCD and TEE had high 
sensitivity and specificity in diagnosis of PFO. The sensitivity of CTTE was too high and led to many false positive cases. Therefore, the diagnostic value of CTCD and TEE was better than CTTE.

\section{Declarations}

Funding: This work was supported by Wenzhou Science and Technology Bureau. (grant numbers Y20190433).

Conflicts of interest: All the authors declare no conflicts of interest.

\section{References}

1. Fisher DC, Fisher EA, Budd JH, Rosen S, E,Goldman ME (1995) The incidence of patent foramen ovale in 1,000 consecutive patients. A contrast transesophageal echocardiography study. Chest 107(6):1504-1509. DOI:10.1378/chest.107.6.1504

2. Overell JR, Bone I, Lees KR (2000) Interatrial septal abnormalities and stroke: a meta-analysis of case-control studies. Neurology 55(8):1172-1179. DOI:10.1212/wnl.55.8.1172

3. Lechat P, Mas JL, Lascault G, Loron P, Theard M, Klimczac M, Drobinski G, Thomas D, Grosgogeat Y (1988) Prevalence of patent foramen ovale in patients with stroke. N Engl J Med 318(18):11481152. DOI:10.1056/nejm 198805053181802

4. Sathasivam S, Sathasivam S (2013) Patent foramen ovale and migraine: what is the relationship between the two? J Cardiol 61(4):256-259. DOI:10.1016/j.jjcc.2012.12.005

5. Schwedt TJ, Demaerschalk BM, Dodick DW (2008) Patent foramen ovale and migraine: a quantitative systematic review. Cephalalgia 28(5):531-540. DOI:10.1111/j.1468-2982.2008.01554.x

6. RESPECT 10-year data strengthens case for PFO closure after cryptogenic stroke. 2015; Available from: http: //www. medscape. com/viewarticle/852810

7. Messé SR, Gronseth GS, Kent DM, Kizer JR, Homma S, Rosterman L, Carroll JD, Ishida K, Sangha N, Kasner SE (2020) Practice advisory update summary: Patent foramen ovale and secondary stroke prevention: Report of the Guideline Subcommittee of the American Academy of Neurology. Neurology 94(20):876-885. DOI:10.1212/wnl.0000000000009443

8. Mitchell C, Rahko PS, Blauwet LA, Canaday B, Finstuen JA, Foster MC, Horton K, Ogunyankin KO, Palma RA, Velazquez EJ (2019) Guidelines for Performing a Comprehensive Transthoracic Echocardiographic Examination in Adults: Recommendations from the American Society of Echocardiography. J Am Soc Echocardiogr 32(1):1-64. DOI:10.1016/j.echo.2018.06.004

9. Yang Y, Guo ZN, Wu J, Jin H, Wang X, Xu J, Feng J, Xing Y (2012) Prevalence and extent of right-toleft shunt in migraine: a survey of 217 Chinese patients. Eur J Neurol 19(10):1367-1372.

DOI:10.1111/j.1468-1331.2012.03793.x

10. Yang J, Zhang H, Wang Y, Zhang S, Lan T, Zhang M, Li Y, Huang W, Zhang H, Wang A, Xiao Y (2020) The Efficacy of Contrast Transthoracic Echocardiography and Contrast Transcranial Doppler for the 
Detection of Patent Foramen Ovale Related to Cryptogenic Stroke. 2020 1513409. DOI: $10.1155 / 2020 / 1513409$

11. Chen J, Chen L, Hu W, Ni X, Zhang Z, Feng X, Fan Z, Chen C, Qiu F, Shao B (2019) A comparison of contrast transthoracic echocardiography and contrast transcranial Doppler in cryptogenic stroke patients with patent foramen ovale. 9 (5): e01283. DOI: 10.1002/brb3.1283

12. Kuijpers T, Spencer FA, Siemieniuk RAC, Vandvik PO, Otto CM, Lytvyn L, Mir H, Jin AY, Manja V, Karthikeyan G, Hoendermis E, Martin J, Carballo S, O'Donnell M, Vartdal T, Baxter C, Patrick-Lake B, Scott J, Agoritsas T, Guyatt G (2018) Patent foramen ovale closure, antiplatelet therapy or anticoagulation therapy alone for management of cryptogenic stroke? A clinical practice guideline. Bmj, 362 k2515. DOI: 10.1136/bmj.k2515

13. Takaya Y, Watanabe N, Ikeda M, Akagi T, Nakayama R, Nakagawa K, Toh N, Ito H (2020) Importance of Abdominal Compression Valsalva Maneuver and Microbubble Grading in Contrast Transthoracic Echocardiography for Detecting Patent Foramen Ovale. J Am Soc Echocardiogr 33(2):201-206. DOI:10.1016/j.echo.2019.09.018

14. Freeman JA, Woods TD (2008) Use of saline contrast echo timing to distinguish intracardiac and extracardiac shunts: failure of the 3- to 5-beat rule. Echocardiography 25(10):1127-1130. DOI:10.1111/j.1540-8175.2008.00741.x

\section{Figures}




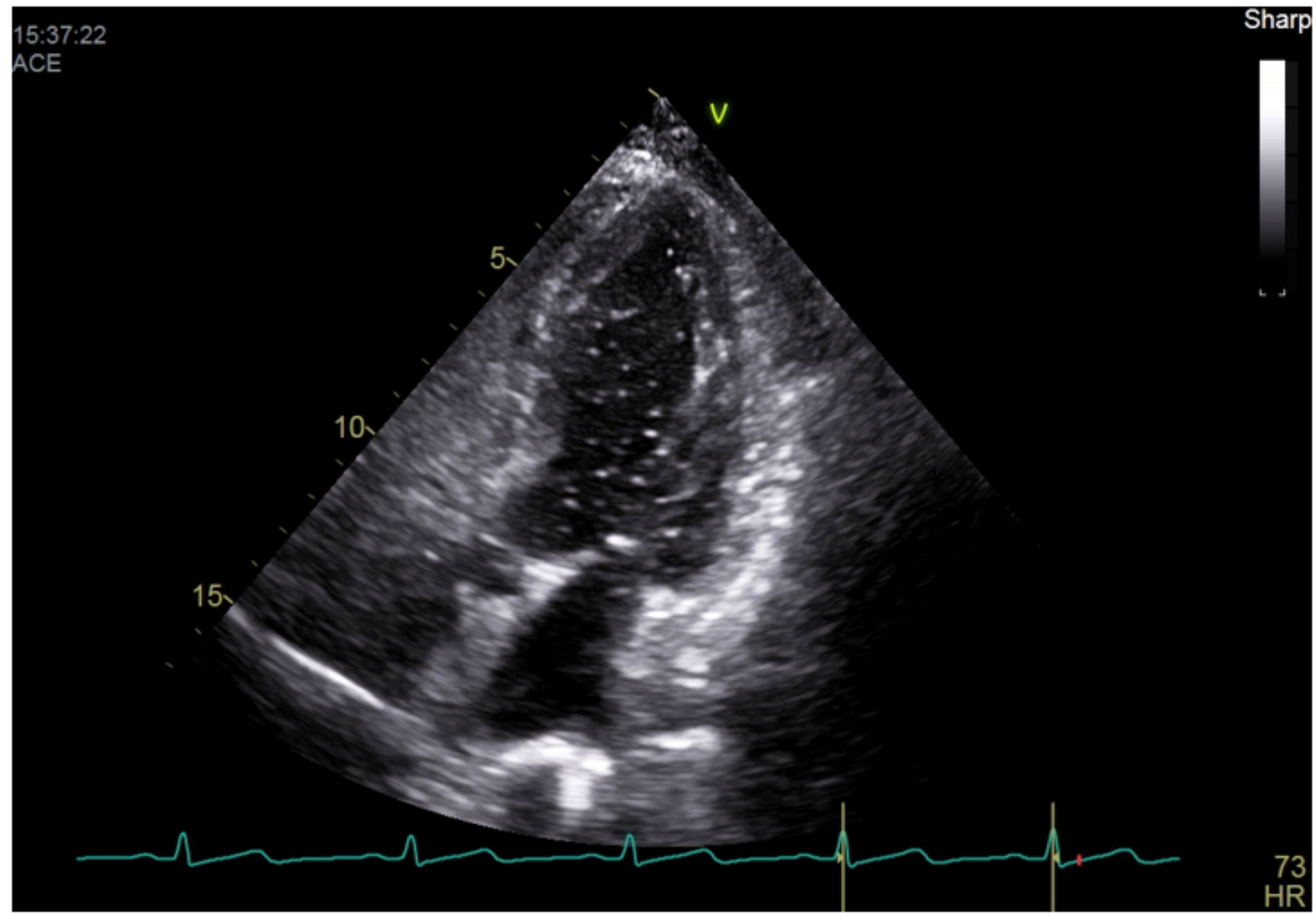

\section{Figure 1}

Contrast transthoracic echocardiography showing massive microbubbles in left ventricle. 


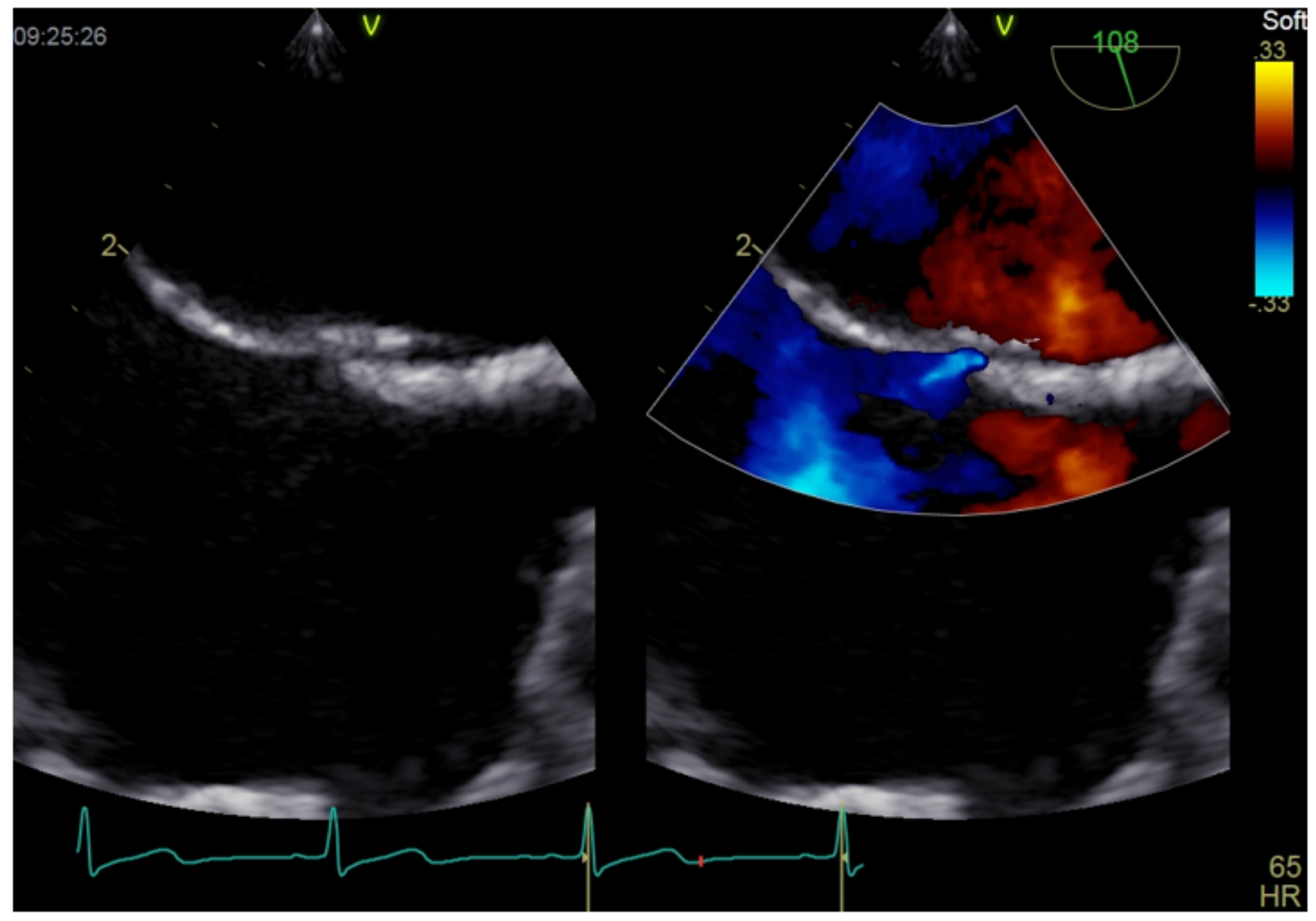

\section{Figure 2}

Transesophageal echocardiography showing along-tunnel PFO and oblique transseptal shunt with Doppler flow echo. 


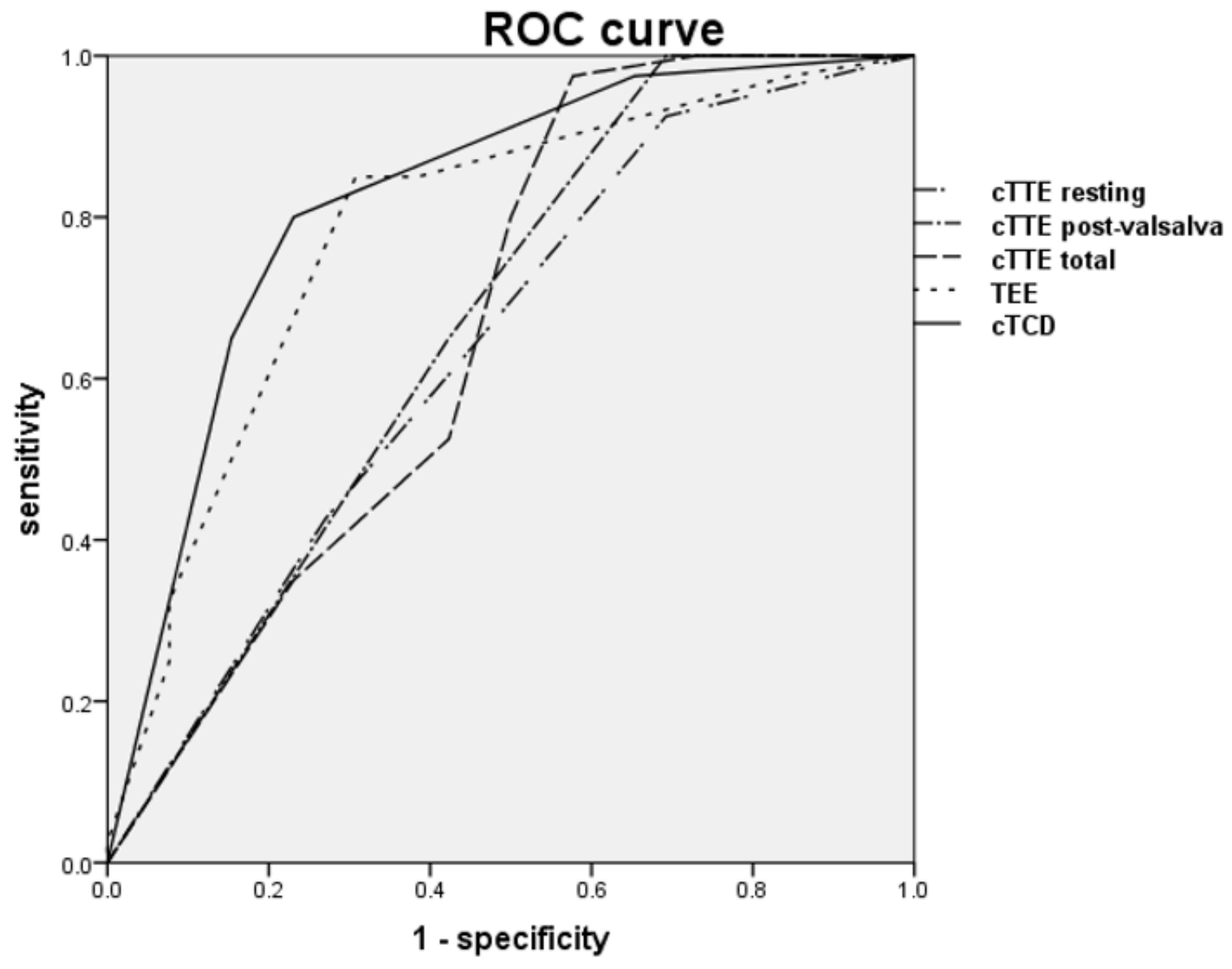

Figure 3

The ROC curve of cTTE $₫$ cTCDITEE 